DOI: 10.4274/jarem.galenos.2021.3803

J Acad Res Med 2021;11(1):62-68

\title{
Could Chest Computed Tomography Scores Assess the Inflammatory Markers and Disease Severity of Coronavirus Disease-2019 Patients?
}

\author{
(D) Yasemin Kayadibi1, (D) Neşe Uçar2, (D) Mehmet Fatih Kaya2, (D) Okan Gürkan², (D) Yeşim Namdar Akan², \\ (D) Berna Demirok 3 \\ ${ }^{1}$ istanbul University-Cerrahpaşa, Cerrahpaşa Faculty of Medicine, Department of Radiology, İstanbul, Turkey \\ 2University of Health Sciences Turkey, Gaziosmanpaşa Training and Research Hospital, Clinic of Radiology, İstanbul, Turkey \\ ${ }^{3}$ University of Health Sciences Turkey, Gaziosmanpaşa Training and Research Hospital, Department of Infection Disease, İstanbul, Turkey
}

Cite this article as: Kayadibi Y, Uçar N, Kaya MF, Gürkan O, Namdar Akan Y, Demirok B. Could Chest Computed Tomography Scores Assess the Inflammatory Markers and Disease Severity of Coronavirus Disease-2019 Patients?. J Acad Res Med 2021;11(1):62-68

\begin{abstract}
Objective: This study aimed to investigate the relationship between chest computed tomography (CT) scores and inflammatory markers in patients with coronavirus disease-2019 (COVID-19).

Methods: A total of 259 patients who were confirmed to be COVID-19 positive with polymerase chain reaction test and CT findings, between 20 March and 30 May 2020, were included in our study. The patients were divided into two groups as critical and moderate according to their clinical conditions. CT findings, complete blood account and C-reactive protein (CRP) were recorded and statistically analysed. Receiver operating characteristic (ROC) curves were plotted in terms of CT scores, white blood cells, neutrophil/lymphocyte ratio (NLR) and CRP for the patients in the moderate and critical groups.

Results: CT scores $(p<0.001)$, neutrophil counts $(p<0.001)$, NLR $(p<0.001)$ and CRP values $(p<0.001)$ were significantly higher in the critical group than in the moderate group, whereas the lymphocyte count $(p<0.001)$ and haemoglobin values $(p=0.003)$ were significantly lower. In the ROC curve analysis, when the cut-off value was set at 6.5 to differentiate the moderate and critical groups, the CT score sensitivity was 0.86 , specificity 0.809 , area under the curve 0.899 and accuracy 0.81 .

Conclusion: There is a correlation between CT scores and NLR. It is crucial to differentiate patients in critical and moderate conditions for the treatment planning of COVID-19. In addition to clinical findings and inflammatory markers, radiological imaging is an effective method to identify patients and determine the prognosis.
\end{abstract}

Keywords: Chest computed tomography, COVID-19, neutrophil/lymphocyte ratio, CT score

\section{INTRODUCTION}

The World Health Organization (WHO) China Country Office first reported cases of pneumonia with unknown origin in Wuhan, China, on 31 December 2020. In the following days, it was announced that the cause of pneumonia is a new coronavirus that has not been previously detected in humans, and the disease was named as coronavirus disease-2019 (COVID-19) (1-3). Because of its rapid transmission from person to person, COVID-19 has

ORCID IDs of the authors: Y.K. 0000-0003-1590-5382; N.U. 0000-0003-2233-4338; M.F.K. 0000-0003-1948-1951; O.G. 0000-0002-7934-9154; Y.N.A. 0000-0003-3957-4661; B.D. 0000-0001-6811-7078.

Corresponding Author/Sorumlu Yazar: Yasemin Kayadibi, E-mail: ysmnkayadibi@gmail.com
Received Date/Geliş Tarihi: 01.10.2020 Accepted Date/Kabul Tarihi: 16.02.2021

(C) Copyright 2021 by University of Health Sciences Turkey, Gaziosmanpaşa Training and Research Hospital. Available on-line at www.jarem.org 
crossed the borders of China and caused a pandemic, and according to WHO's COVID-19 epidemiologic update in January 2021, it has infected 96 million people worldwide and caused 2 million deaths.

The factor of COVID-19 mostly affects the respiratory system in humans, and the spectrum of the illness severity ranges from a simple cold to severe respiratory failure $(2,4-6)$. It has been reported that it may involve enteric, hepatic, nephrotic and neurological systems as well as the respiratory system (4-6).

The diagnostic reference of COVID-19 is the examination of the obtained respiratory tract samples by reverse-transcription polymerase chain reaction (PCR) $(2,3)$. However, insufficient sampling, sampling too early or in the late phase of infection and sampling with inappropriate method may cause false-negative results (6). In possible/definite COVID-19 cases, chest computed tomography (CT) is used to support diagnosis, demonstrate lung involvement and evaluate the extent of lung infection (710). The most frequent $C T$ findings of patients are peripherally located ground-glass density and vascular thickening, and their CT findings may be positive, although the PCR test results are negative $(4,8,10,11)$. In a recent study, the sensitivity of chest CT (98\%) was higher than that of the PCR test (10).

There are four clinical stages of the disease [early stage (days: 0-4)], progression period (days: 5-8), peak stage (days: 9-13) and resolution phase (after day: 14). Radiological findings may vary according to these stages (11-13). The distinction of the critical group from the non-critical group according to the patients' clinical manifestations is important for treatment choice and prognosis (14-16). There are publications examining the relationship between $\mathrm{CT}$ findings of the disease and its clinical manifestations and prognosis $(10,13-15,17)$. However, studies comparing radiological findings with laboratory findings are limited $(17,18)$. This study aimed to investigate the role of patients' lung involvement score to distinguish between the moderate and critical groups of patients and to assess which laboratory markers are correlated with the chest $\mathrm{CT}$ involvement score.

\section{METHODS}

This retrospective study was approved by the Local Ethics Committee University of Health Sciences Turkey, Gaziosmanpaşa Training and Research Hospital (approval number: 89, approval date: 28.05.2020) and the Republic of Turkey Ministry of Health, COVID-19 Scientific Research Committee. Written informed consent was obtained from the patients enrolled. Between 20 March and 30 May 2020, 330 patients between 18 and 90 years old who were admitted to our hospital with suspicion of COVID-19 pneumonia and underwent chest CT were retrospectively reviewed using picture archiving communication systems. Of these patients, those with positive COVID-19 PCR test results and positive COVID-19 CT images with appropriate imaging protocol $(n=259)$ were included in the study. Those who had negative PCR test results and had no evidence of typical COVID-19 pneumonia $(n=71)$ were excluded from the study. A total of 259 patients were included in the study. Laboratory findings, complete blood count (number/ $\mu \mathrm{L}$ ) and CRP $(\mathrm{mg} / \mathrm{dL})$, presence of comorbidities [hypertension, diabetes mellitus (DM), heart failure, renal failure, malignancy history and chronic respiratory diseases] at the first hospital admission and clinical findings of the patients were obtained from the hospital archive. Care was taken to ensure that the laboratory data and CT images were acquired on the same day at the time of admission.

In terms of their clinical features at the time of hospital admission, patients were divided into two groups: moderate and critical laccording to the guideline on COVID-19 (trial version 5) issued by the China National Health Commission] (19). The moderate group was composed of patients with fever, cough or shortness of breath only, whereas the critical group comprised those with Tachypnea ( $>30$ breaths per minute), with less than $93 \%$ oxygen saturation at room environment or with $\mathrm{PaO}_{2} / \mathrm{FiO}_{2}<300 \mathrm{mmHg}$ and with mechanical ventilation and intensive care requirement.

All images were acquired using a multidetector scanner (GE Optima CT660, 64 slices; General Electric, USA, 5 mm collimation) with the following parameters: $120 \mathrm{kVp}, 40 \mathrm{~mm}$ beam collimation, 5 $\mathrm{mm}$ image thickness, $500 \mathrm{~mm}$ reconstruction field of view, $500 \mathrm{~mA}$ (maximum), $10 \mathrm{~mA}$ (minimum) and $1 \mathrm{~s}$ rotation time. Images were evaluated by two radiologists, "Yasemin Kayadibi and Neşe Uçar" with 9 and 11 years of chest radiology experiences, respectively. Image examination parameters such as ground-glass opacity, consolidation, vascular thickening, interlobular septal thickening, mediastinal lymphadenopathy, pleural effusion, subpleural bands and pericardial effusion were evaluated. CT images were scored from 1 to 4 (1: $0 \%-25 \%$; 2: $26 \%-50 \%$; 3: 51\%-75\%; 4: 76\%-100\%) for each lobe according to the semiquantitative scoring system described in the literature $(9,10,14,15)$. Scores determined for all lobes were summed, and the total CT score (from 0 to 20) was determined by the consensus of the two radiologists (Yasemin Kayadibi and Neşe Uçar).

\section{Statistical Analysis}

The statistical software SPSS version 22.0 IBM was used for data analysis. As descriptive analyses, frequency rates and percentages were described for the categorical variables and means and standard deviations for the continuous variables. Normality was checked using the Shapiro-Wilk test. The MannWhitney $U$ and Student's t-tests were used for non-normally and normally distributed variables, respectively. Receiver operating characteristic (ROC) was used to determine the cut-off values, sensitivity and specificity. The chi-square test was used to test relationships between categorical variables. A p-value of $<0.05$ was considered statistically significant. For the correlation analysis, the Spearman test was used as a non-parametric test.

\section{RESULTS}

\section{Patients Analysis}

Table 1 presents patient analysis details. A total of 259 patients were included in the study. Patients whose clinical and laboratory 


\begin{tabular}{|l|l|l|l|}
\hline \multicolumn{3}{|l}{ Table 1. Demographic features of patients } \\
\hline Parameters & $\begin{array}{l}\text { Moderate } \\
(\mathbf{n = 1 7 3 )}\end{array}$ & $\begin{array}{l}\text { Critical } \\
(\mathbf{n = 8 6})\end{array}$ & $\begin{array}{l}\text { Total } \\
(\mathbf{n}=\mathbf{2 5 9 )}\end{array}$ \\
\hline Mean age (years) & 53.2 & 63.18 & 56.52 \\
\hline Sex (n, \%) & & & \\
\hline Men & $80(46.2)$ & $57(66.3)$ & $122(47)$ \\
\hline Women & $93(53.8)$ & $29(33.7)$ & $137(53)$ \\
\hline Comorbidities (n, \%) & $117(67.6)$ & $86(100)$ & $208(80)$ \\
\hline Hypertension & $50(43)$ & $33(38.4)$ & $85(41)$ \\
\hline Diabetes mellitus & $30(25.6)$ & $21(24.4)$ & $53(25.5)$ \\
\hline Heart disease & $11(9.4)$ & $12(14)$ & $24(11.5)$ \\
\hline Respiratory disorders & $19(16)$ & $8(9.3)$ & $27(13)$ \\
\hline Renal dysfunction & $5(4.3)$ & $9(10.4)$ & $14(6.7)$ \\
\hline Malignancy & $2(1.7)$ & $3(3.5)$ & $5(0.9)$ \\
\hline
\end{tabular}

findings could not be reached, who had negative $P C R$ results and those with tomography images having artefacts were excluded in the study. Of patients, 122 (80 moderate and 57 critical) were men and 137 (93 moderate and 29 critical) women. Men dominated the critical group $(p=0.02)$, whereas no gender dominated the moderate group. The mean ages of patients were 53.2 and 63.18 years in moderate and critical groups, respectively. Comorbidity was present in 208 of 259 patients.

\section{Radiological Findings}

All CT images were obtained at the time of patients' first hospital admission. The summary of radiological and laboratory findings of the patients is presented in Table 2. Considering the radiological findings, a comparison of the two groups revealed that the lower lobes were statistically significantly higher in the severe group in which the disease affected more than one lobe. There was no significant difference in terms of right and left lung involvement in both groups $(p=0.684)$. The right lower lobe was the most commonly involved lobe (93\%). There was no significant difference between the number of lobes involved in both groups $(p=0.152)$. The detection rate of ground-glass opacity was similar in both groups ( $p=0.792)$; however, the prevalence of consolidation and crazy paving were higher in the critical group ( $p<0.001$ for both). Central involvement between the two groups was significantly higher in the critical group $(p<0.001)$. Pleural effusion and subpleural band formation were observed significantly higher in the critical group $(p<0.001$ and $p=0.005$, respectively). The total mean CT score of the moderate and critical groups were 4.8 and 10.8, respectively. The CT score in the critical group was significantly higher than in the moderate group according to the Mann-Whitney $U$ test $(p<0.001)$. ROC curve analysis revealed that for a cut-off value of 6.5 , the CT score had a sensitivity of 0.86 , specificity 0.809 , area under the curve (AUC) 0.899 and accuracy 0.81 (with 95\% confidence interval: 0.854-0.944; Figure 1, 2).

\section{Laboratory Findings}

Table 3 presents the curves obtained from laboratory findings. The mean white blood cell (WBC) value was 6,845 in the total patient group, 6,335 in the moderate group and 8,335 in the critical group. WBC values were statistically significant $(p<0.001)$ between the critical and moderate groups according to the MannWhitney $U$ test after normality evaluation using the Shapiro-Wilk test ( $p<0.001)$. Increased neutrophil count, decreased lymphocyte count and increased CRP amount were statistically significantly higher in the critical group $(p<0.001)$ according to the chisquare test. There was no significant difference in haemoglobin and platelet levels between the groups $(p=0.003$ and 0.265 , respectively). Neutrophil/lymphocyte ratio (NLR) was statistically significantly higher in the critical group than in the moderate group ( $p<0.001$ ) according to the Mann-Whitney $U$ test.

\section{Evaluation of the Relation between CT Scores and Laboratory Findings}

When the correlation between CT scores and laboratory findings was compared, it was found that there was a positive correlation between WBC ( $r=0.341)$, neutrophil count ( $r=0.457)$, NLR ( $r=496)$, CRP ( $r=0.750)$ and CT scores, whereas there was a negative correlation between CT scores and lymphocyte counts ( $r=-0.315)$. The highest correlation was found between CRP and CT scores (Figure 3, Table 4). Spearman test was used for correlation analysis because of the value of $p<0.001$ obtained as a result of the normality calculation performed using the Shapiro-Wilk test.

\section{DISCUSSION}

Chest CT plays an important role in the diagnosis and follow-up of COVID-19 treatment (10). Many studies examine the relationship between the CT findings of the disease and its clinical manifestation $(7,12,16,20,21)$. In the literature, areas of ground-glass density, crazy-paving pattern and vascular congestion, which are the most common findings observed in COVID-19 pneumonia, are predicted to reflect the virus-related septal and alveolar damage, the less frequently observed consolidations reflect the exudate in alveoli and hyaline membrane formation. A limited number of autopsy studies also support these findings (13,20-22). Similarly, in our study, the most common finding observed in the whole patient population was ground-glass density areas involving more than one lobe, but consolidations were statistically significantly higher in the critical group.

In COVID-19 pneumonia, a better prognosis is expected in the moderate group, whereas worse prognosis is likely in the critical group. It has been shown that early distinction between the two groups and early treatment has increased the survival rates significantly. CT scoring systems were developed to calculate the radiological involvement of the disease, and a significant correlation was found between the high score, clinical conditions and laboratory findings $(8,12,15,17,18,20)$. In our study, we aimed to investigate the relationship between lung involvement severity and inflammatory markers in patients diagnosed with COVID-19 and the role of CT score in differentiating clinical severity. Li et al. (20), Liu et al. (15) and Francone et al. (17) reported the cut-off values for CT scores at 7, 4 and 18, respectively, to distinguish the moderate and critical groups. In our study $(n=259)$, this value was 


\section{Table 2. Summary of chest CT and laboratory findings}

\begin{tabular}{|c|c|c|c|c|}
\hline & Moderate $(n=173)$ & Critical $(n=86)$ & Total $(n=259)$ & $p$ \\
\hline \multicolumn{5}{|l|}{ Chest $C T$ findings } \\
\hline Right upper lobe (n, \%) & $131(76)$ & $82(95)$ & $213(82)$ & $<0.001^{\star \star}$ \\
\hline Right middle lobe (n, \%) & $109(63)$ & $81(94)$ & $190(73)$ & $<0.001^{\star \star}$ \\
\hline Right lower lobe (n, \%) & $160(93)$ & $82(95)$ & $242(93)$ & 0.439 \\
\hline Left upper lobe (n, \%) & $132(76)$ & $79(92)$ & $211(82)$ & $0.002^{\star \star}$ \\
\hline Left lower lobe (n, \%) & $145(84)$ & $84(98)$ & $229(88.4)$ & $0.001^{\star \star}$ \\
\hline Single lobe (n, \%) & $12(6.9)$ & $2(2)$ & $14(5.4)$ & 0.152 \\
\hline Multiple lobes (n, \%) & $161(93)$ & $84(98)$ & $245(95)$ & 0.152 \\
\hline Total CT score (mean \pm SD) & $4.8 \pm 0.17)$ & $10.8 \pm 0.45)$ & $6.8 \pm 4.12)$ & $<0.001 * *$ \\
\hline GGD (n, \%) & $168(97)$ & $84(98)$ & $252(97)$ & 0.792 \\
\hline Consolidation (n, \%) & $65(38)$ & $57(66)$ & $122(47)$ & $<0.001^{\star *}$ \\
\hline Crazy-paving pattern (n, \%) & $39(23)$ & $46(54)$ & $85(33)$ & $<0.001^{* *}$ \\
\hline Round (n, \%) & $131(76)$ & $64(74)$ & $195(75)$ & 0.879 \\
\hline Patchy (n, \%) & $104(60)$ & $76(88)$ & $180(70)$ & $0.001^{\star *}$ \\
\hline Peripheric distribution ( $n, \%)$ & $153(88.4)$ & $86(100)$ & $239(92)$ & $0.001^{\star \star}$ \\
\hline Central distribution (n, \%) & $64(37)$ & $67(78)$ & $131(51)$ & $0.001 * \star$ \\
\hline Peripheral + central (n (\%)) & $60(35)$ & $67(78)$ & $127(49)$ & $0.001 * \star$ \\
\hline Single focus (n, \%) & $12(7)$ & $0(0)$ & $12(4.6)$ & $0.01 *$ \\
\hline Multiple foci (n, \%) & $161(93)$ & $86(100)$ & $247(95.4)$ & $0.01 *$ \\
\hline Halo sign (n, \%) & $38(22)$ & $19(22)$ & $57(22)$ & 0.981 \\
\hline Reverse halo sign (n, \%) & $14(8)$ & $9(10.5)$ & $23(9)$ & 0.643 \\
\hline Vascular thickening (n, \%) & $51(29.5)$ & $34(40)$ & $85(34)$ & 0.123 \\
\hline Pleural effusion (n, \%) & $1(0.6)$ & $12(14)$ & $13(5)$ & $0.001^{\star \star}$ \\
\hline Lymph node enlargements (n, \%) & $7(4)$ & $7(8)$ & $14(5.4)$ & 0.241 \\
\hline Subpleural bants (n, \%) & $37(21.4)$ & $33(38)$ & $70(27)$ & $0.005^{\star}$ \\
\hline Three in bud appearance (n, \%) & $7(4)$ & $6(7)$ & $13(5)$ & 0.367 \\
\hline Dilatation of peripheral airways ( $n, \%)$ & $20(12)$ & $15(17.4)$ & $35(14)$ & 0.246 \\
\hline Fibrous stripe (n, \%) & $31(18)$ & $22(25.6)$ & $53(21)$ & 0.190 \\
\hline \multicolumn{5}{|l|}{ Laboratory findings } \\
\hline WBC count (mean \pm SD) & $6.1 \pm 0.21)$ & $8.3 \pm 0.39)$ & $6.85 \pm 3.22)$ & $<0.001^{\star *}$ \\
\hline Increased WBC (n, \%) & $7(4)$ & $19(22)$ & $26(10)$ & $<0.001^{\star \star}$ \\
\hline Neutrophil count (mean \pm SD) & $4.14 \pm 0.18)$ & $6.84 \pm 0.38)$ & $5.04 \pm 3.09)$ & $<0.001^{* *}$ \\
\hline Increased neutrophil (n, \%) & $8(4.6)$ & $25(29)$ & $33(13)$ & $<0.001^{\star *}$ \\
\hline Lymp count (mean \pm SD) & $1.41 \pm 0.05)$ & $1.6 \pm 0.39)$ & $1.5 \pm 2.1)$ & $<0.001^{\star *}$ \\
\hline Decreased lymp (n, \%) & $45(26)$ & $47(55)$ & $92(36)$ & $<0.001^{\star *}$ \\
\hline NLR & $3.53 \pm 0.21)$ & $8.74 \pm 0.94)$ & $5.3 \pm 6.02)$ & $<0.001^{\star *}$ \\
\hline Platelet count (mean \pm SD) & $197.59 \pm 5.47)$ & $212.57 \pm 9.99)$ & $202.56 \pm 79.39)$ & 0.256 \\
\hline Increased platelet (n, \%) & $2(1.2 \%)$ & $2(2 \%)$ & $4(1.5 \%)$ & 0.602 \\
\hline $\mathrm{Hb}($ mean $\pm \mathrm{SD})$ & $133.65 \pm 1.32)$ & $126.93 \pm 1.82)$ & $131.42 \pm 17.48)$ & $0.003^{*}$ \\
\hline Decreased Hb (n, \%) & $12(7)$ & $12(14)$ & $24(9)$ & 0.073 \\
\hline $\mathrm{CRP}($ mean $\pm \mathrm{SD})$ & $58.74 \pm 10.97)$ & $135 \pm 13.49)$ & $84.07 \pm 142.53)$ & $<0.001^{\star *}$ \\
\hline İncreased CRP (n, \%) & $146(84.4)$ & $86(100)$ & $232(90)$ & $<0.001 * *$ \\
\hline
\end{tabular}

The $p$-values for $C T$ scores and WBC were calculated using the Mann-Whitney $U$ test. The $p$-values for other parameters were calculated using the Pearson chisquare test. ${ }^{*} P<0.05,{ }^{* *} p<0.001$.

CT: computed tomography, GGD: ground-glass appearance, WBC: white blood cells, NLR: neutrophil/lymphocyte ratio, CRP: C-reactive protein, Lymp: lymphocyte, Hb: haemoglobin, SD: standard deviation 


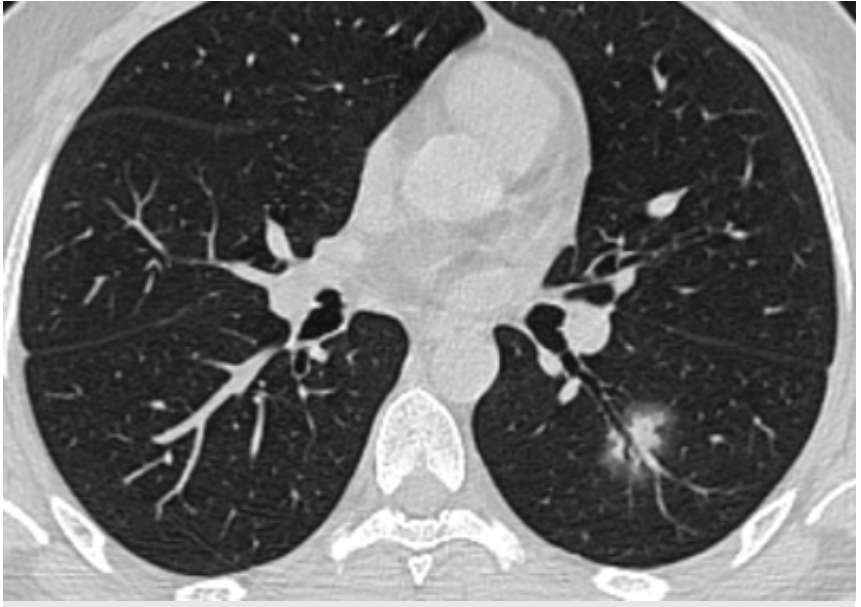

Figure 1. Chest computed tomography (CT) example of the moderate group: a 30-year-old man (CT score: 1, neutrophil/ lymphocyte ratio: $2.5, \mathrm{C}$-reactive protein: 3.5 ). The axial CT image revealed a single focus of ground-glass opacity with adjacent dilated bronchus

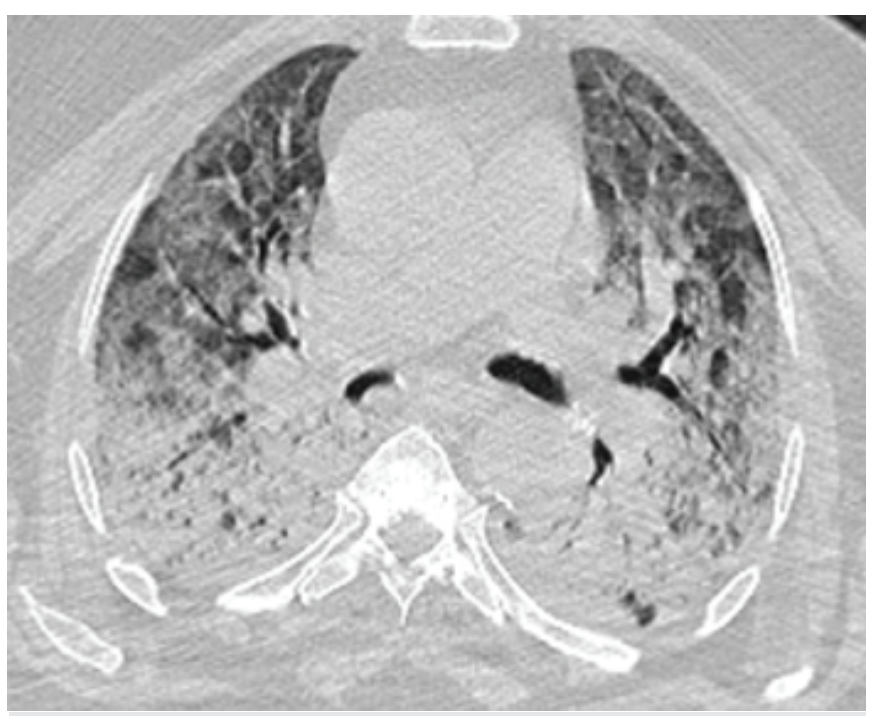

Figure 2. Chest computed tomography (CT) example of the critical group: A 79-year-old man (CT score: 19, neutrophil/ lymphocyte ratio: 12.5, C-reactive protein: 190). Axial CT image revealed bilateral diffuse ground-glass opacities and consolidations with dilated bronchi and air bubbles
6.5 (AUC: 0.899, specificity: 0.809, sensitivity: 0.86 and accuracy: 0.81). In addition to the clinical and laboratory data of the patient, this study has demonstrated that chest CT findings are also useful in the distinction of disease severity.

Many alterations occur in the immune and haematopoietic systems of patients diagnosed with COVID-19. In the literature, various laboratory findings including lymphopenia and elevated liver enzymes, lactate dehydrogenase, inflammatory markers (e.g. CRP and ferritin), D-dimer (>1 mcg/mL), prothrombin time, troponin and creatine phosphokinase have been associated with poor prognosis in COVID-19 $(14,15,17,20)$. Immune cell destruction, impaired immune cell function, decreased lymphocyte level as a result of bone marrow involvement and simultaneous neutrophil dominance are the most striking findings in the haematological system in COVID-19 (22-25). NLR, as a marker of inflammatory response, has been associated with poor prognosis in sepsis, cardiovascular diseases and malignancies (4,14,23-25). Fu et al. (26) found a significant difference between the mild/moderate and severe groups in terms of WBC, NLR, lymphocyte counts, D-dimer levels and fibrinogen levels. Among these parameters, the highest AUC was 0.88 , which was the most significant parameter for NLR. They also argued that the change in haematological parameters is an earlier and more reliable change than inflammatory markers (26). The study by Lagunas-Rangel (27) also supported the statistically significant increase in NLR in severe patients.

In our study, the CRP level, neutrophil count and NLR in the critical group increased statistically significantly than in the moderate group ( $p<0.001$ for all), whereas the lymphocyte count decreased statistically significantly $(p<0.001)$. In the ROC curve analysis, it was calculated as $47.9 \mathrm{mg} / \mathrm{dL}$ (AUC: 0.812) for CRP, $6.13 \mathrm{n} / \mu \mathrm{L}$ (AUC: 0.777) for WBC, $4.38 \mathrm{n} / \mu \mathrm{L}$ (AUC: 0.757) for neutrophil count, $1.04 \mathrm{n} / \mu \mathrm{L}$ (AUC: 0.683) for lymphocyte count and 3.75 (AUC: 0.792) for NLR. In addition, a positive correlation was noted between CT scores and CRP, WBC, neutrophil count and NLR and a negative correlation with lymphocyte count (correlation coefficients: 0.496 , $0.341,0,457,0.496$ and -0.315 , respectively). Our study showed that the CT score in patients' initial tomography was significantly related to the patient's inflammatory response and NLR. Our study is also compatible with studies in the literature that reveal the correlation between CRP, D-dimer, NLR and decreased lymphocyte count and CT scores $(17,18,25-27)$.

Table 3. Cut-off values and statistical analysis in distinguishing the moderate and critical groups

\begin{tabular}{|l|l|l|l|l|l|}
\hline & Cut-off value & AUC (Cl: lower-upper) & Specificity & Sensitivity & Accuracy \\
\hline Total $C T$ Score & 6.5 & $0.899(0.854-0.944)$ & 0.809 & 0.86 & 0.81 \\
\hline WBC $(n / \mu L)$ & 6.13 & $0.712(0.646-0.779)$ & 0.601 & 0.686 & 0.629 \\
\hline Neu $(n / \mu L)$ & 4.38 & $0.773(0.712-0.834)$ & 0.659 & 0.709 & 0.675 \\
\hline Lymp $(n / \mu L)$ & 1.04 & $0.683(0.613-0.752)$ & 0.593 & 0.699 & 0.664 \\
\hline NLR & 3.74 & $0.792(0.729-0.855)$ & 0.74 & 0.802 & 0.76 \\
\hline CRP & 47.9 & $0.812(0.758-0.866)$ & 0.682 & 0.802 & 0.722 \\
\hline
\end{tabular}

CT: computed tomography, WBC: white blood cells, Neu: neutrophil, NLR: neutrophil/lymphocyte ratio, CRP: C-reactive protein, Lymp: lymphocyte, AUC: areas under the curve, $\mathrm{Cl}$ : confidence interval 
generally conducted by chest radiography. Patients who have positive PCR with no radiological findings, have negative PCR with positive radiological findings and did not require hospitalisation were excluded in the study, and therefore, we may have caused bias in patient selection in our study.

\section{CONCLUSION}

The demographic data of our study are compatible with other studies investigating the correlation between CT scores and NLR among the studies conducted to date are limited in number. Many clinical parameters are used to determine the critical patient group in COVID-19 pneumonia. In our study, we showed that the findings observed in chest $\mathrm{CT}$ reflect the severity of the disease at the time of admission. We found a positive correlation between CT scores and NLR, which has a prognostic side, and a determined cut-off score of 6.5 to distinguish the moderate and critical groups. We think that it can guide clinicians to determine the severity of involvement and choose an effective treatment according to the severity of the disease.

Ethics Committee Approval: This retrospective study was approved by the Local Ethics Committee University of Health Sciences Turkey, Gaziosmanpaşa Training and Research Hospital (approval number: 89, approval date: 28.05.2020).

Informed Consent: Written informed consent was obtained from the patients enrolled.

Peer-review: Externally peer-reviwed.

Author Contributions: Surgical and Medical Practices - B.D.; Concept N.U.; Design - Y.K.; Data Collection and/or Processing - N.U., M.F.K., O.G.; Analysis and/or Interpretation - Y.K.; Literature Search - Y.N.A.; Writing Manuscript - Y.K.

Conflict of Interest: The authors have no conflict of interest to declare.

Figure 3. Receiver operating characteristic analysis for (a) computed tomography score, (b) white blood cells, (c) neutrophil count, (d) lymphocyte count, (e) neutrophil/lymphocyte ratio and (f) C-reactive protein

ROC: Receiver operating characteristic

Although COVID-19 is known to cause infection in completely healthy adults, more serious involvement is observed in adults with advanced age and comorbidities such as cardiovascular diseases, DM, hypertension, chronic lung diseases, cancer and chronic kidney disease $(15,17,18,21,23)$. In our study, the comorbidity rate was higher in the critical group than in the moderate group, and the most common comorbidity was hypertension, followed by DM.

\section{Study Limitations}

Our study has some limitations. Our study was single-centred and retrospective. There are four clinical stages of the disease [early stage (days: 0-4), progression period (days: 5-8), peak stage (days: 9-13) and resolution phase (after day: 14)], and radiological findings may vary according to these stages. However, in our study, it was impossible to obtain the information from the patients' records about which date the patients were admitted to the hospital after the onset of the disease and at which stage of the disease the chest CT was obtained. Except for patients who did not worsen, control follow-up CT was not requested, and follow-up was
Financial Disclosure: The authors declared that this study has received no financial support.

\section{REFERENCES}

1. Wang C, Horby PW, Hayden FG, Gao GF. A novel coronavirus outbreak of global health concern. Lancet 2020; 395: 470-3.

2. Zhu N, Zhang D, Wang W, Li X, Yang B, Song J, et al. A Novel Coronavirus from Patients with Pneumonia in China, 2019. N Eng J Med 2020; 382: 727-33.

3. Lu R, Zhao X, Li J, Niu P, Yang B, Wu H, et al. Genomic characterisation and epidemiology of 2019 novel coronavirus: implications for virus origins and receptor binding. Lancet 2020; 395: 565-74.

4. Wang D, Hu B, Hu C, Zhu F, Liu X, Zhang J, et al. Clinical characteristics of 138 hospitalized patients with 2019 novel coronavirus-infected pneumonia in Wuhan, China. JAMA 2020; 323: 1061-9.

5. Clinical characteristics of 2019 novel coronavirus infection in China | medRxiv [Internet]. [cited 2020 Jun 26]. Available from: https://www. medrxiv.org/content/10.1101/2020.02.06.20020974v1

6. Tan W, Zhao X, Ma X, Wang W, Niu P, Xu W, et al. A novel coronavirus genome identified in a cluster of pneumonia Cases - Wuhan, China 2019-2020. China CDC Weekly 2020; 2: 61-2.

7. Kim H. Outbreak of novel coronavirus (COVID-19): What is the role of radiologists? Eur Radiol 2020; 30: 3266-7

8. Pan $Y$, Guan $H$, Zhou $S$, Wang $Y$, Li Q, Zhu T, et al. Initial CT findings and temporal changes in patients with the novel coronavirus pneumonia (2019-nCoV): a study of 63 patients in Wuhan, China. Eur Radiol 2020; 30: 3306-9.

9. Chan JFW, Yuan S, Kok KH, To KKW, Chu H, Yang J, et al. A familial cluster of pneumonia associated with the 2019 novel coronavirus indicating 
person-to-person transmission: a study of a family cluster. Lancet 2020; 395: 514-23.

10. Fang $Y$, Zhang $H$, Xie J, Lin M, Ying L, Pang $P$, et al. Sensitivity of Chest $C T$ for COVID-19: comparison to RT-PCR. Radiology 2020; 296: E115-7. doi: 10.1148/radiol.2020200432.

11. Ooi GC, Khong PL, Müller NL, Yiu WC, Zhou LJ, Ho JCM, et al. Severe acute respiratory syndrome: temporal lung changes at thin-section $\mathrm{CT}$ in 30 patients. Radiology 2004; 230: 836-44.

12. Pan $Y$, Guan $H$, Zhou $S$, Wang $Y, L i ~ Q$, Zhu T, et al. Initial CT findings and temporal changes in patients with the novel coronavirus pneumonia (2019-nCoV): a study of 63 patients in Wuhan, China. Eur Radiol. 2020; 30: 3306-9.

13. Zheng F, Tang W, Li H, Huang YX, Xie YL, Zhou ZG. Clinical characteristics of 161 cases of corona virus disease 2019 (COVID-19) in Changsha. Eur Rev Med Pharmacol Sci 2020; 24: 3404-10.

14. Onder G, Rezza G, Brusaferro S. Case-fatality rate and characteristics of patients dying in relation to COVID-19 in Italy. JAMA 2020; 323: 1775-6.

15. Liu J, Chen T, Yang H, Cai Y, Yu Q, Chen J, et al. Clinical and radiological changes of hospitalised patients with COVID-19 pneumonia from disease onset to acute exacerbation: a multicentre paired cohort study. Eur Radiol 2020; 30: 5702-8.

16. Wang J, Liu J, Wang Y, Liu W, Chen $X$, Sun $C$, et al. [Dynamic changes of chest CT imaging in patients with corona virus disease-19 (COVID-19)]. Zhejiang Da Xue Xue Bao Yi Xue Ban 2020; 49: 191-7.

17. Francone M, lafrate F, Masci GM, Coco S, Cilia F, Manganaro L, et al. Chest CT score in COVID-19 patients: correlation with disease severity and short-term prognosis. Eur Radiol 2020; 30: 6808-17.

18. Tan C, Huang Y, Shi F, Tan K, Ma Q, Chen Y, et al. C-reactive protein correlates with computed tomographic findings and predicts severe COVID-19 early. J Med Virol 2020; 92: 856-62.
19. New Coronavirus Pneumonia Diagnosis and Treatment Plan (Trial Version 5) Interpretation of the government website of the State Administration of Chinese Medicine. Last Accessed Date: 27.01.2021. Available from: http://bgs.satcm.gov.cn/zhengcewenjian/2020-02-06/12848.html

20. Li K, Wu J, Wu F, Guo D, Chen L, Fang Z, et al. The clinical and chest CT features associated with severe and critical COVID-19 pneumonia. Invest Radiol 2020; 55: 327-31.

21. Chung $M$, Bernheim A, Mei X, Zhang N, Huang $M$, Zeng $X$, et al. CT Imaging Features of 2019 Novel Coronavirus (2019-nCoV). Radiology 2020; 295: 202-7.

22. XuZ, Shi L, Wang Y, Zhang J, Huang L, Zhang C, et al. Pathological findings of COVID-19 associated with acute respiratory distress syndrome. Lancet Respir Med 2020; 8: 420-2.

23. Zhou $F, Y u T, D u R$, Fan G, Liu $Y$, Liu $Z$, et al. Clinical course and risk factors for mortality of adult inpatients with COVID-19 in Wuhan, China: a retrospective cohort study. Lancet 2020; 395: 1054-62.

24. Shi S, Qin M, Shen B, Cai Y, Liu T, Yang F, et al. Association of cardiac injury with mortality in hospitalized patients with COVID-19 in Wuhan, China. JAMA Cardiol 2020; 5: 802-10.

25. Huang $Y$, Liu A, Liang L, Jiang J, Luo $H$, Deng $W$, et al. Diagnostic value of blood parameters for community-acquired pneumonia. Int Immunopharmacol 2018; 64: 10-5.

26. Fu J, Kong J, Wang W, Wu M, Yao L, Wang Z, et al. The clinical implication of dynamic neutrophil to lymphocyte ratio and D-dimer in COVID-19: a retrospective study in Suzhou China. Thromb Res 2020; 192: 3-8.

27. Lagunas-Rangel FA. Neutrophil-to-lymphocyte ratio and lymphocyte-toC-reactive protein ratio in patients with severe coronavirus disease 2019 (COVID-19): A meta-analysis. J Med Virol 2020; 92: 1733-4. 